\title{
Temporal Effects of Bisphenol A on Dopaminergic Neurons: An Experiment on Adult Rats
}

\author{
Masami Ishido ${ }^{*}, 1$ and Yoshinori Masuo ${ }^{2}$ \\ ${ }^{I}$ Center for Environmental Risk Research, National Institute for Environmental Studies, Japan \\ ${ }^{2}$ Toho University, Laboratory of Neuroscience, Department of Biology, Faculty of Science, Chiba, Japan
}

\begin{abstract}
The vulnerability of developing brains to environmental chemicals has been reported. Here, we examined the temporal effects of bisphenol A on dopaminergic neurons. Exposure of 5-day-old rats to bisphenol A caused hyperactivity in juveniles, after which spontaneous motor activity leveled off at 8 weeks of age, when apoptotic cell death was still observed along with the loss of tyrosine hydroxylase immunoreactivity. Accumulation of $\alpha$-synuclein, a pathological marker of neurodegeneration, was also detectable in the substantia nigra, but not in the cerebral cortex. In adult rats, acute toxicity following microinjection of bisphenol $\mathrm{A}(20 \mu \mathrm{g})$ caused degeneration of dopaminergic neurons, similar to that following administration of 6-hydroxydopamine $(15 \mu \mathrm{g})$. Chronic exposure of adult rats to bisphenol A ( $3 \mathrm{mg} / \mathrm{kg} / \mathrm{day}$ for 28 days) caused neurodegeneration in the substantia nigra. However, behavioral effects were not seen after either acute or chronic exposure of adult rats to bisphenol A.
\end{abstract}

Thus, the sensitivity of the central nervous system to bisphenol A was shown to be different at different life stages, confirming the greater vulnerability of the developing central nervous system.

Keywords: Bisphenol A, endocrine disruptors, neurodegeneration, vulnerability.

\section{INTRODUCTION}

Bisphenol A [2,2-bis(4-hydroxyphenyl)propane] is a plasticizer that is widely used to produce polycarbonate plastic, epoxy resin, and unsaturated polystyrene. The widespread use of bisphenol A has resulted in the frequent exposure to the compound of the majority of the population of the developed world. Circulating levels of bisphenol A measured in humans appear sufficient to induce detrimental effects [1].

An emerging body of evidence is accumulating that bisphenol A exerts effects on the central nervous system (CNS), in addition to its effects on the reproductive system [2]. Since animals are very sensitive to exogenous chemicals during perinatal or neonatal periods, many animal studies have focused on these times [3]. We previously have demonstrated, in neonatal (5-day-old) rats, the toxicity of bisphenol A for dopaminergic neurons by exposure to the chemical $[4,5]$. Other studies have shown that developmental exposure to bisphenol A might alter the dopaminergic system at the pre- or post-synaptic level [6]. This is a rapidly expanding area of research [7].

Dopamine is a highly conserved neurotransmitter involved in a number of complex functions such as regulation of movements, goal-directed behavior, cognition, attention, and reward-seeking behavior [8]. Progressive degeneration of dopamine neurons is seen in Parkinson's

*Address correspondence to this author at the Center for Environmental Risk Research, National Institute for Environmental Studies, 16-2 Onogawa, Tsukuba 305-8506, Japan; Tel: +81-29(850)-2396; Fax: +81-29(850)-2870; E-mail: ishidou@nies.go.jp disease, which is estimated to affect $2-3 \%$ of the population more than 65 years of age [9-11]. Clinically, the cardinal symptoms are bradykinesia, resting tremor, rigidity, and postural instability. Adult animal models of Parkinson's disease have been generated by selective dopaminergic degeneration with neurotoxins such as 6-hydroxydopamine (6-OHDA), 1-methyl-4-phenyl-1,2,3,6-tetrahydropyridine (MTPT), or rotenone $[12,13]$.

Recently, in addition to developmental exposure to bisphenol $\mathrm{A}$, there have been reported about exposure of adult animals to the chemical [14-16]. The current study was designed to examine whether exposure of adult rats to bisphenol A would cause neurodegeneration, particularly of dopaminergic neurons, resulting in a model of Parkinson's disease.

\section{MATERIALS AND METHODS}

\subsection{Chemicals}

Bisphenol A was purchased from Wako Chemical Corporation (Tokyo, Japan). 6-Hydroxydopamine hydrobromide was purchased from Sigma-Aldrich (St. Louis, Missoruri, USA). Olive oil was purchased from Nacalai Chemical Corporation (Kyoto, Japan).

\subsection{Neonatal Animals and Treatments with Bisphenol $\mathbf{A}$}

Pregnant Wistar rats were obtained from Clea Japan (Tokyo, Japan), and were maintained for at least 1 week, housed in home cages, and fed with standard laboratory chow (MF diet, Oriental Yeast Corp., Tokyo, Japan) and distilled water ad libitum, at $22{ }^{\circ} \mathrm{C}$, in a light-dark cycle $(12 \mathrm{~h} / 12 \mathrm{~h})$. Typically, about 50 male pups were born from 10 
pregnant rats, and 5-7 pups were randomly housed. They were weaned at 3 weeks of age. This study was carried out in strict accordance with the experiment and related activities in Academic Research Institutions guidelines, under jurisdiction of the Ministry of Education, Culture, Sports, Science and Technology. The protocol was approved by the Committee on the Ethics of Animal Experiments of the National Institute for Environmental Studies.

Bisphenol A was suspended in a minimal amount of 50\% ethanol and made up to volume with olive oil. The chemical ( $87 \mathrm{nmol}$ equivalent to $20 \mu \mathrm{g} / 10 \mu \mathrm{l} / \mathrm{rat}$ ) was intracisternally injected into 5-day-old pups. Control rats were injected with vehicle alone $(10 \mu 1)$.

\subsection{Measurements of Spontaneous Motor Activity}

Spontaneous motor activity of rats was individually measured in a home cage using a Supermex system (Muromachi Kikai, Japan), as previously described [17]. In this system, a sensor detects and measures the radiated body heat of an animal. A Supermex sensor head consists of paired infrared pyroelectric detectors. This system detects any object with a temperature at least $5{ }^{\circ} \mathrm{C}$ higher than background within a cone-shaped area having a $6 \mathrm{~m}$ diameter and a $110^{\circ}$ vertex. The sensor monitors motion in multiple zones of the cage through an array of Fresnel lenses placed above the cage, and movement of the animal in the $X, Y$, and $Z$ axes can be determined. Activity was measured every $15 \mathrm{~min}$ for 22-24 h while animals were maintained in a $12 \mathrm{~h}$ light:dark cycle. Food and water were provided ad libitum at the beginning of the measurement period, and rats were not subsequently disturbed in any way. The activity of 10 rats was concurrently recorded.

\subsection{Adult Animal Surgery}

For stereotaxic microinjection of chemicals, all surgery was performed as described previously [18]. Male 9-weekold rats were anesthetized with ketamine $(150-175 \mathrm{mg} / \mathrm{kg}$ intraperitoneally) and placed on a stereotaxic apparatus with the incisor bars adjusted to $3.3 \mathrm{~mm}$ above the interaural line. A 25 -gauge $(0.5 \mathrm{~mm}$ OD) stainless-steel blunt-tipped syringe needle connected to a Hamilton microsyringe $(10 \mu \mathrm{l})$ by means of Teflon tubing was inserted into the left substantia nigra; the coordinates were $\mathrm{AP}=+3.0 \mathrm{~mm}$ from the interaural line, $\mathrm{L}=1.8 \mathrm{~mm}$ and $\mathrm{H}=+2.1 \mathrm{~mm}$, calculated according to the atlas [19]. Bisphenol A (20 $\mu \mathrm{g})$ or 6-OHDA $(15 \mu \mathrm{g})$ was injected into the substantia nigra over a 3-min period using a microsyringe mounted on a Syringe-Infusion Pump 22 (Harvard Apparatus, Holliston, Massachusetts, USA). The needle was left in place for $3 \mathrm{~min}$ before being slowly withdrawn. For the sham operation, $3 \mu \mathrm{l}$ of vehicle was injected under the conditions described.

For chronic exposure to chemicals, Alzet osmotic mini pumps (model 2ML4 or 2ML1, DURECT Corp., Cupertino, California, USA) were used as previously described [20]. The pumps were filled with chemicals dissolved in equal volumes of dimethylsulfoxide (DMSO) and polyethylene glycol (PEG). Pumps were then placed in sterile $0.9 \%$ saline at $37^{\circ} \mathrm{C}$ for at least $4 \mathrm{~h}$, and then were implanted under the skin on the back of each animal. Control rats received pumps filled with DMSO:PEG (1:1) only.

\subsection{Rotation Test}

After 2 weeks, rats stereotaxically injected with or without chemicals were subjected to the rotation test. The rats received methamphetamine $(3 \mathrm{mg} / \mathrm{kg}$ intraperitoneally) and then rotation behavior was monitored with a video tape recorder (Sony model PC120, Tokyo, Japan) for $10 \mathrm{~min}$ and was scored.

\subsection{Immunohistochemistry}

Immunostaining was carried out as described previously [4]. Rats were sacrificed by decapitation at the ages indicated. Whole brain samples were fixed in $10 \%$ phosphate-buffed formalin ( $\mathrm{pH}$ 7.2) and embedded in paraffin. Sagittal and coronal sections $(5 \mu \mathrm{m})$ were mounted on siliconized slides (Matsunami, Kishiwada, Japan), deparaffinized and hydrated. Each section was permealized with $0.5 \%$ Triton X-100 in phosphate-buffered saline (PBS) and blocked with $10 \%$ normal horse serum plus $4 \%$ bovine serum albumin for $30 \mathrm{~min}$ at $4{ }^{\circ} \mathrm{C}$. Samples were incubated with the primary antibodies described below in the presence of $4 \%$ bovine serum albumin and either $0.05 \%$ Triton X-100, tyrosine hydroxylase (1:100; Sigma-Aldrich), glutamic-acid decarboxylase 65/67 (1:100; Sigma-Aldrich), or $\alpha$-synuclein (1:100; MP Biomedicals Inc.). After three washes with PBS containing $0.1 \%$ Tween 20 , each sample was incubated with secondary fluorescence-labeled goat anti-mouse or -rabbit immunoglobulin G (IgG; 1:2,000; Sigma-Aldrich) or biotinylated goat anti-rabbit $\operatorname{IgG}(1: 2,000$; Lab Vision Corp., Fremont, California, USA), for $60 \mathrm{~min}$ at room temperature. Each fluorescence-labeled sample was washed twice with PBS and observed under a microscope (IX70; Olympus, Tokyo, Japan); the biotinylated samples were labeled with streptavidin-conjugated alkaline phosphatase and visualized with 3,3'-diaminobenzidine (Fig. 4) or 5-bromo-4-chloro-3indolyl phosphate/nitroblue tetrazolium (Fig. 5). Images were captured using Viewfinder Lite version 1.0 camera software and a DP-50 digital camera (Olympus).

\subsection{In Situ TUNEL Labeling}

In situ TUNEL labeling was performed using an Apoptotag In Situ Cell Death Detection kit (Intrerigene, Purchase, NY, USA) as described previously, with slight modifications [5]. The whole brain sample was fixed in $10 \%$ phosphate-buffered formalin $(\mathrm{pH} 7.2)$ and embedded in paraffin. After deparaffinization in xylene and rehydration through a graded series of ethanol dilutions, the sample was treated with $20 \mu \mathrm{g} / \mathrm{ml}$ of proteinase $\mathrm{K}$ for $15 \mathrm{~min}$ at room temperature. After washing twice with $\mathrm{PBS}, \mathrm{H}_{2} \mathrm{O}_{2}(2 \%)$ was used to quench the endogenous peroxidase. The sample was then incubated with equilibration buffer for $15 \mathrm{~s}$, terminal deoxynucleotidyl transferase reaction solution for $1 \mathrm{~h}$ at $37^{\circ} \mathrm{C}$ and Reaction Stop buffer for $10 \mathrm{~min}$. It was then blocked in $4 \%$ serum and $0.1 \%$ Triton X-100 in PBS for 30 min with intermittent washes in PBS. The sample was incubated with a peroxidase-conjugated sheep antidigoxigenin antibody overnight at $4{ }^{\circ} \mathrm{C}$. Finally, it was processed for the visualization of immunoreactive protein using 3,3'-diaminobenzidine as the substrate for the color reaction. Images were captured as described above. 


\subsection{Fluoro-Jade Staining}

Brain tissues from control and bisphenol A-exposed rats were stepwise rehydrated with decreasing concentrations of alcohol, as described above. Fluoro-Jade C histochemistry was done according to the manufacturer's protocol (CHEMICON, Temecula, California, USA).

\subsection{Statistics}

Statistical analyses were carried out with repeated measure ANOVA or the Student's $t$ test using StatView Ver. 5.0 software (SAS Institute, Cary, NC, USA).

\section{RESULTS}

\subsection{Behavioral Traits of Adult Rats with Neonatal Bisphenol A Lesions}

Previously, we demonstrated that intracisternal administration of $20 \mu \mathrm{g}$ of bisphenol A (equivalent to $87 \mathrm{nmol}$ in $10 \mu \mathrm{l}$ of olive oil per rat) caused hyperactivity in the juvenile ( $4 \sim 5$ weeks of age), compared to control rats. In the current study, we further measured their spontaneous motor activity at 8 weeks of age. Fig. (1) shows that the bisphenol A-induced hyperactivity seen at $4 \sim 5$ weeks of age had leveled off at 8 weeks of age, suggesting a compensatory input against the dopaminergic neuronal deficit.

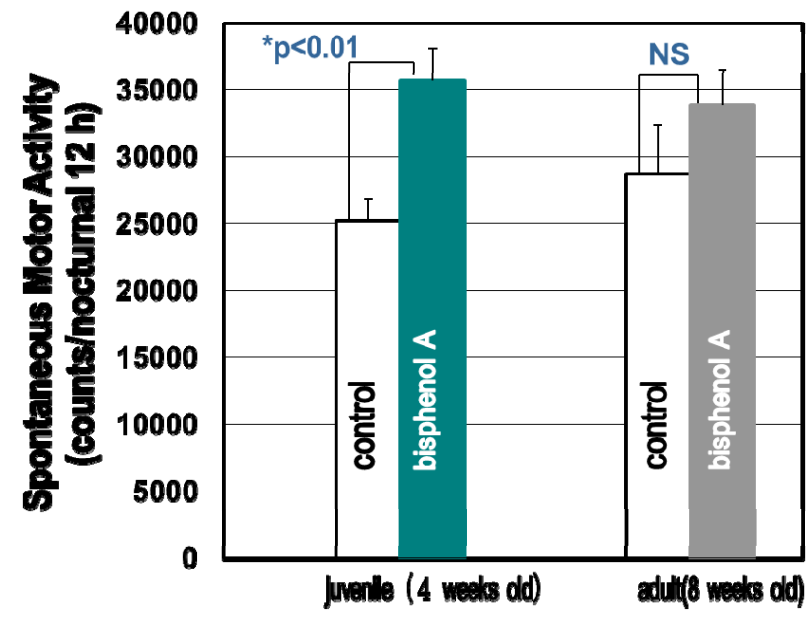

Fig. (1). Effects of neonatal bisphenol A-induced lesions on adult behavior in the rat. Following exposure of 5-day-old pups to bisphenol A $(20 \mu \mathrm{g})$, spontaneous motor activity was measured during dark periods at 4 weeks (left) and at 8 weeks (right) of age. Control pups received vehicle alone. ${ }^{*} \mathrm{P}<0.01$. NS: not significant.

\subsection{Long-Term Effects in Adult Rats of Neonatal Bisphenol A Treatment}

We then investigated the long-term effects of bisphenol A using immunohistochemical analyses. Brain tissues from 8-week-old rats with neonatal bisphenol A lesions were processed for immunoreactivity to tyrosine hydroxylase, which is a rate-limiting enzyme in catecholamine synthesis. In the control rats, the substantia nigra or ventral tegmental area of the brain tissue strongly stained with the anti-tyrosine hydroxylase antibody (Fig. 2a). However, a single intracisternal injection of bisphenol A $(20 \mu \mathrm{g})$ into rat pups resulted in a large reduction of tyrosine hydroxylase immunoreactivity in the substantia nigra pars compacta and the ventral tegmental area at 8 weeks of age (Fig. 2b); this indicated degeneration of dopaminergic neurons. Similar results were observed following a single intracisternal injection of $100 \mu \mathrm{g}$ of 6-OHDA (data not shown). Immunostaining for glutamic-acid decarboxylase, which is involved in $\gamma$-aminobutyric-acid synthesis, revealed its presence in the substantia nigra pars reticulata (Fig. 2c, d). This confirmed that the effects of bisphenol A were specific to dopaminergic neurons.

To determine whether the bisphenol A-induced reduction of immunoreactive tyrosine hydroxylase in the mesencephalon reflected degeneration of the dopaminergic neurons, we performed terminal deoxynucleotidyl transferase-mediated dUTP nick end labeling (TUNEL). No labeled cells were observed in the control brain tissue (Fig. 3a). By contrast, TUNEL-positive cells with nuclear condensation were detected in the treated group (Fig. $\mathbf{3 b}, \mathbf{c}$ ), which indicated that bisphenol A induced apoptotic cell death in the substantia nigra.

Furthermore, we performed an immunohistochemical analysis of $\alpha$-synuclein in the chemically lesioned rats, since it is reported to be a key component of the pathological process of neurodegeneration [21]. Fig. (4) shows that the control tissues showed faint staining (Fig. 4a, d), whereas a significant accumulation of $\alpha$-synuclein was seen in the substantia nigra pars compacta of adult rats that had been neonatally treated with bisphenol A (Fig. 4b, c). Furthermore, diffuse staining of $\alpha$-synuclein was observed much more in the cortex of chemically treated rats (Fig. 4e, f) compared with that in the controls (Fig. 4d).

\subsection{Microinjection of Bisphenol A into the Substantia Nigra of Adult Rats}

To determine the acute effects of bisphenol A on adult dopaminergic neurons, we stereotaxically injected bisphenol A $(20 \mu \mathrm{g})$ or 6 -OHDA $(15 \mu \mathrm{g})$ into the left substania nigras of 9-week-old rats. Control rats received vehicle alone. After 2 weeks, the rotation test was carried out after administration of methamphetamine and was monitored using a video tape recorder. The rotation behavior was then quantified (Fig. 5A). The rats with 6-OHDA lesions rotated about 45 times in $10 \mathrm{~min}$, whereas those treated with bisphenol A performed about 1 4 rotations during the same time period. Subsequently, the brain tissues were processed to assess immunoreactivity of striatal tyrosine hydroxylase. The striatum in control rats was strongly stained with the antityrosine hydroxylase antibody (Fig. 5B a). Microinjection with bisphenol A resulted in a large reduction of ipsilateral tyrosine hydroxylase immunoreactivity in the striatum (Fig. 5B b), indicating degeneration of dopaminergic neurons. This was also observed after microinjection of $15 \mu \mathrm{g}$ of 6-OHDA (Fig. 5B c).

\subsection{Chronic Exposure of Adult Rats to Chemicals with Osmotic Pumps}

The chronic effects of bisphenol A on 7-week-old rats were determined using osmotic pumps. At the beginning of exposure, the chemical concentration was $3 \mathrm{mg} / \mathrm{kg} /$ day. The adult rats were exposed to bisphenol A for 28 days. There 

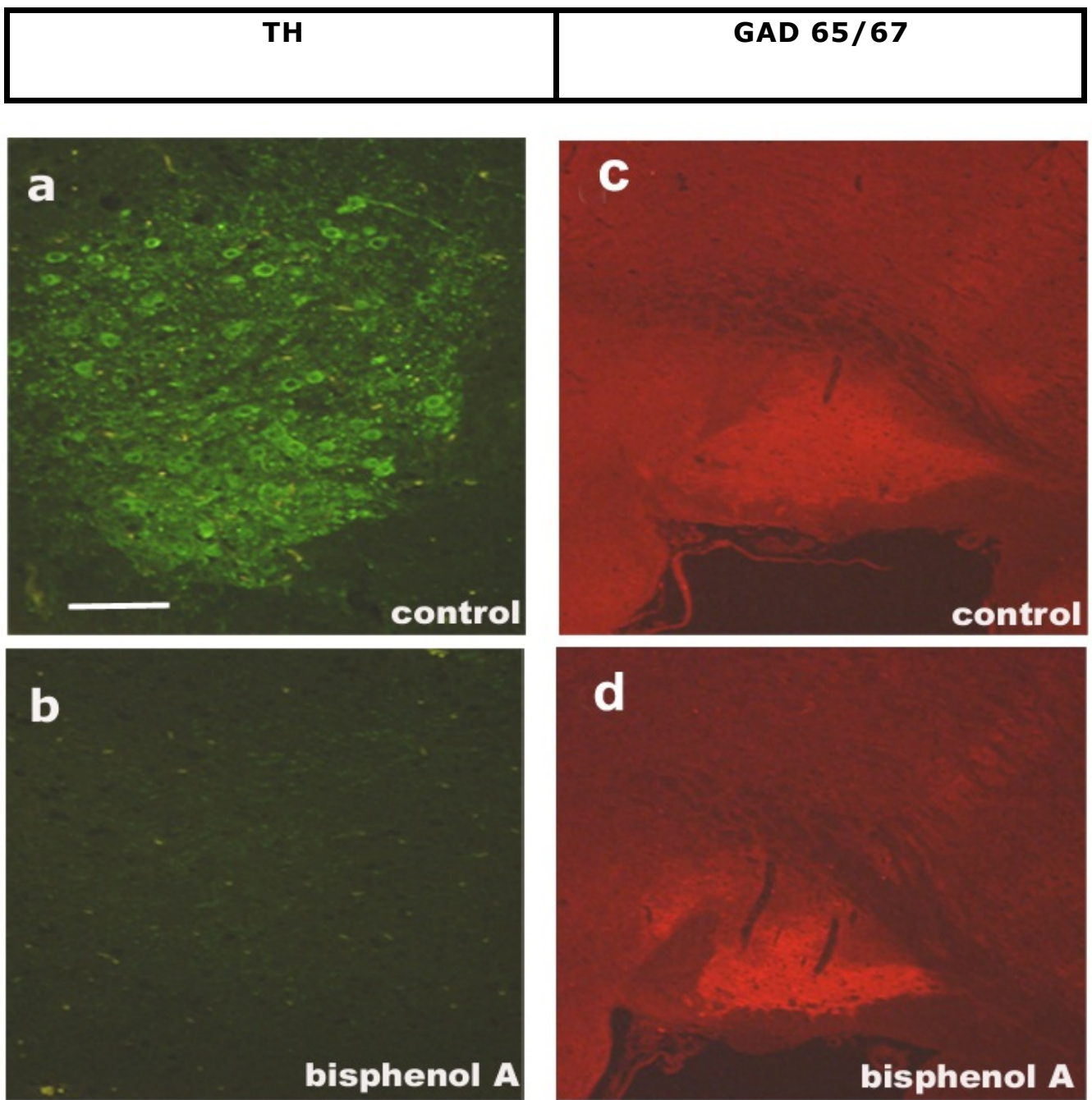

Fig. (2). Selective loss of tyrosine hydroxylase immunoreactivity after bisphenol A administration. After intracisternal injection of vehicle alone (a and $\mathbf{c}$ ) or $20 \mu \mathrm{g}$ bisphenol A (b and d) into 5-day-old rats, sagittal sections of brain at 8 weeks of age were stained with anti-tyrosine hydroxylase antibody (TH; $\mathbf{a}$ and b) or anti-glutamic-acid decarboxylase (GAD 65/67; $\mathbf{c}$ and d). Scale bar $=50 \mu \mathrm{m}$.
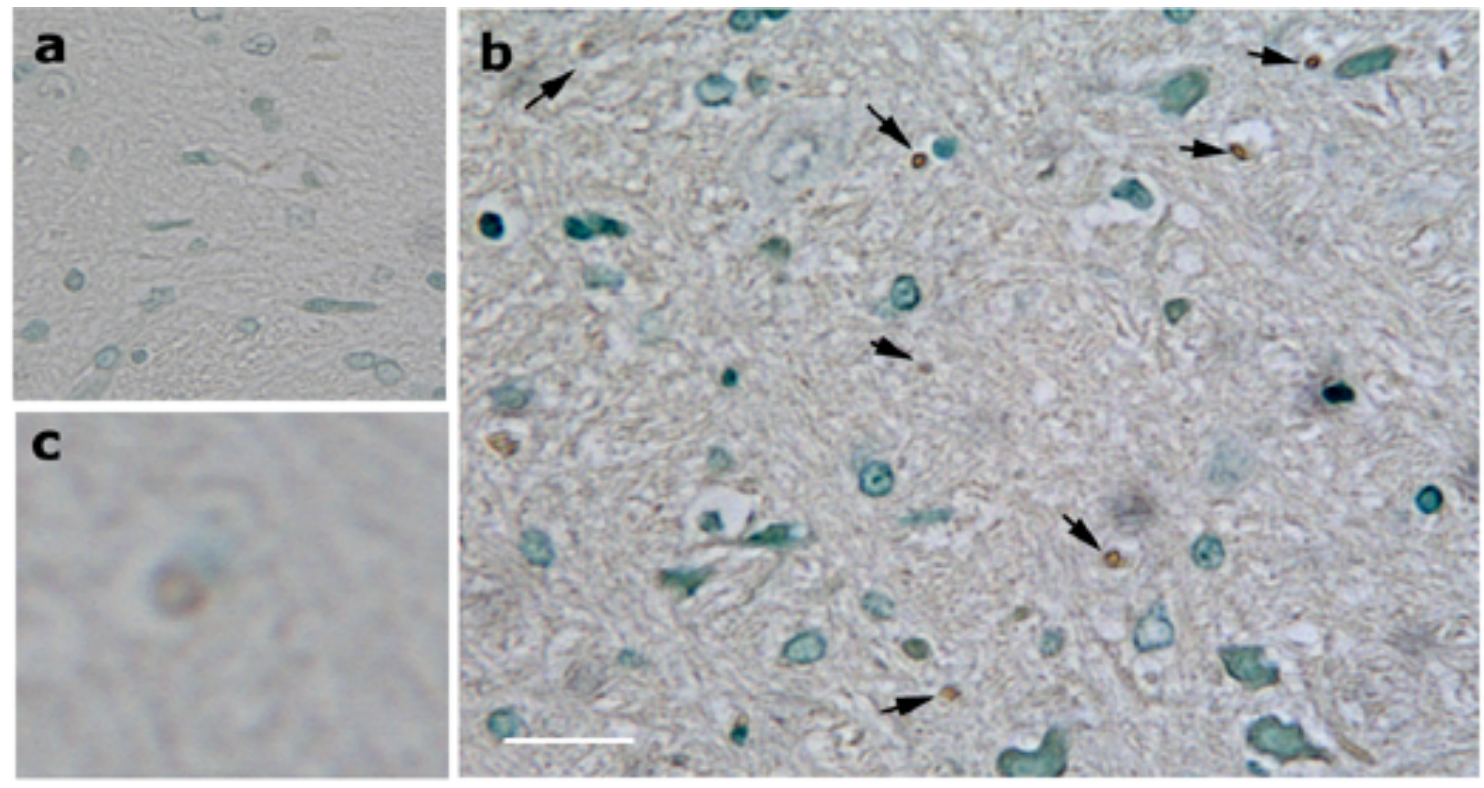

Fig. (3). In situ TUNEL staining of the mesencephalon. After intracisternal injection of (a) vehicle alone or (b) $20 \mu \mathrm{g}$ bisphenol A into 5-day-old rats, sagittal sections of brain at 8 weeks of age were stained with TUNEL. (c) A higher resolution image of apoptotic nuclei (arrow) induced by the chemical. Scale bar $=25 \mu \mathrm{m}$. 

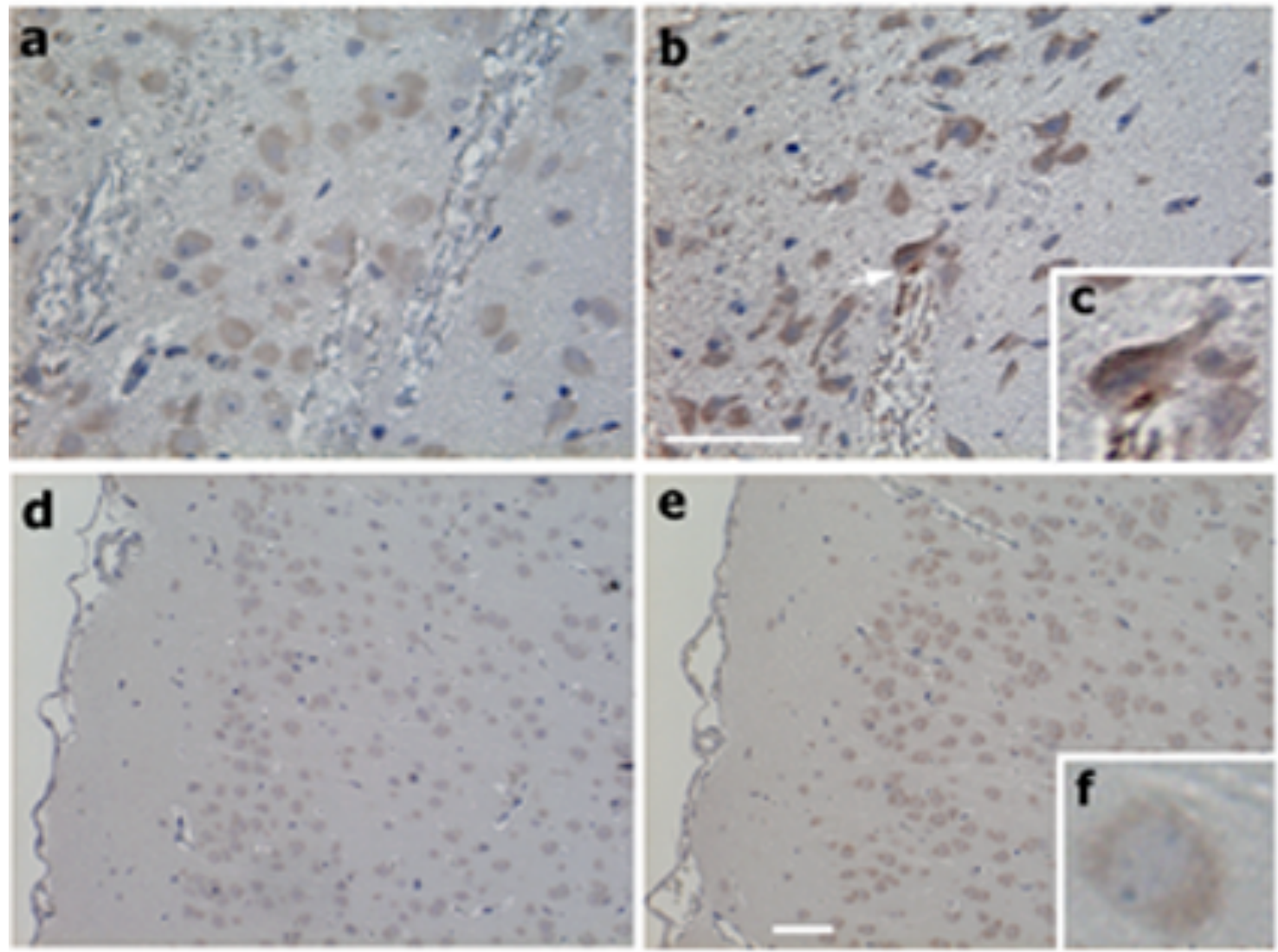

Fig. (4). Pathological hallmarks of neurodegeneration in adult rats lesioned as neonates by bisphenol A administration. After intracisternal injection of vehicle alone (a and $\mathbf{d})$ or $20 \mu \mathrm{g}$ bisphenol A (b and e) into 5-day-old rats, coronal sections of brain at 8 weeks of age were stained with anti- $\alpha$-synuclein antibody $(\mathbf{a}-\mathbf{f})$. The substantia nigra is shown in $\mathbf{a}-\mathbf{c}$, the cortex is shown in $\mathbf{d}-\mathbf{f}$ and higher resolution images (c and $\mathbf{f})$ are shown in the insets. Scale bar $=50 \mu \mathrm{m}$.

was no significant difference in spontaneous motor activity, as shown in Fig. (6A), in contrast to rotenone $3 \mathrm{mg} / \mathrm{kg} / \mathrm{day}$, a dopaminergic toxin, which caused $49 \%$ hypoactivity, as seen in Parkinson's model rats (manuscript in preparation). Brain tissue was then stained with Fluoro-Jade (Fig. 6B); fluorescent signals were observed in the substantia nigra of bisphenol A-treated rats (Fig. 6B b), whereas none were seen in the control rat tissues (Fig. 6B a), indicating neurodegeneration by bisphenol $\mathrm{A}$ in adult rats.

\section{DISCUSSION}

Neuronal toxicity of bisphenol A has been shown in experimental animals. Since animals are very sensitive to exogenous chemicals during perinatal or neonatal periods, many animal studies have focused on these times. The effects of perinatal exposure to bisphenol A have been shown to affect various aspects of behavior such as exploratory, sociosexual [22], and aggressive behaviors [23], as well as anxiety levels [24], and nociception [25], and the response to D-amphetamine [26]. Impairment by bisphenol A of sexual differentiation of exploratory behavior and the size of the locus coeruleus was also observed [27], and it also increased depression-like behavior [28]. Furthermore, neocortical histogenesis was perturbed by bisphenol A [29]. We have demonstrated that rat hyperactivity was elicited by bisphenol A via both intracisternal administration [4] and oral administration to 5-day-old Wistar pups [5].
In this study, we demonstrated neurodegeneration in the adult rat by acute and chronic exposure to bisphenol $\mathrm{A}$. It is notable that bisphenol A degenerates adult dopaminergic neurons. However, it did not lead to behavior alteration, whereas neonatal administration of bisphenol A caused hyperactivity in the juvenile. The extent of lesions in the adult rat was not sufficient to induce behavioral alterations. As seen in Fig. (5B), microinjection of 6-OHDA into the unilateral substantia nigra completely degenerated striatal dopaminergic neurons (c), whereas that of bisphenol A did not completely destroy those neurons (b). Therefore, behavioral effects of bisphenol A were not observed (Fig. 5A). This might reflect the fact that threshold for Parkinson's disease is less than $20 \%$ of remaining striatal dopamine neurons [10]. This might be the case of chronic exposure to bisphenol A (Fig. 6A, B). Thus, the developing central nervous system is much more vulnerable to bisphenol A exposure. This might reflect the fact that the ability of the newborn to metabolize, detoxify and eliminate many toxins is different from that of adults, in the brain as well as in peripheral systems [30]. Furthermore, postnatal neurological development is a highly complex process, involving cell division, differentiation, and migration during structuring of the central nervous system; therefore, this period might present numerous "windows of opportunity" for the chemical to interfere with normal neuronal development. 
(A)

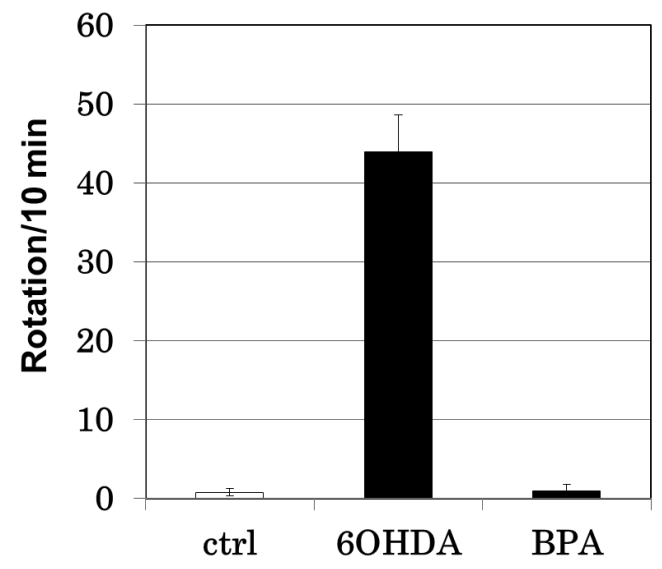

(B)
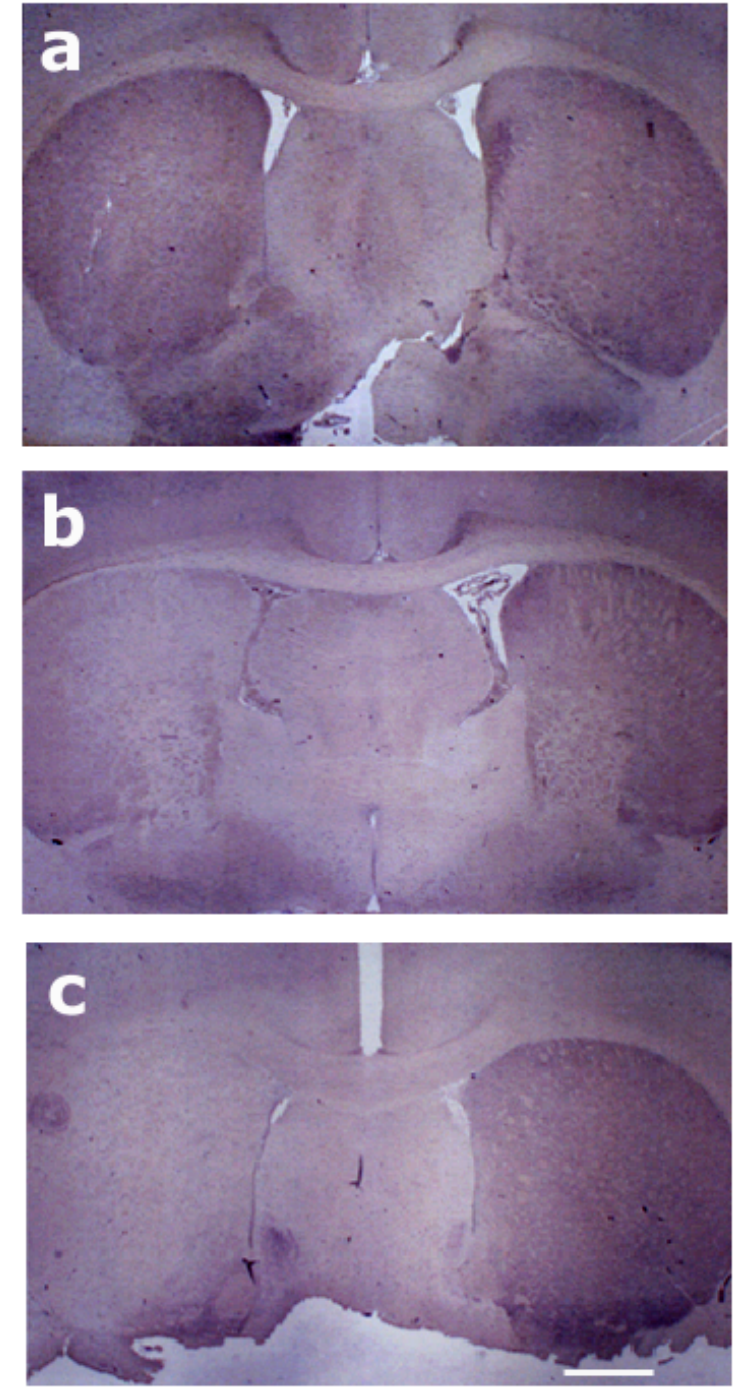

Fig. (5). Microinjection of bisphenol A or 6-hydroxydopamine in the substantia nigra of adult rats. Male 9-week-old rats were anesthetized with ketamine (150-175 mg/kg intraperitoneally) and placed on a stereotaxic apparatus, followed by injection of vehicle (ctrl: $n=9$ ), bisphenol A (BPA: $20 \mu \mathrm{g} ; \mathrm{n}=5$ ) or 6-hydroxydopamine (6OHDA: $15 \mu \mathrm{g}$; $=5$ ) into the substantia nigra over a 3-min period. (A) After 2 weeks, rotation behavior induced by methamphetamine $(3 \mathrm{mg} / \mathrm{kg}$, intraperitoneally) was monitored using a video recorder and was scored. (B) Brains were then removed and subjected to immunohistochemistry to detect striatal tyrosine hydroxylase, as indicated. a: ctrl; b: BPA; c: 6 OHDA. Scale bar $=1 \mathrm{~mm}$. 
(A)

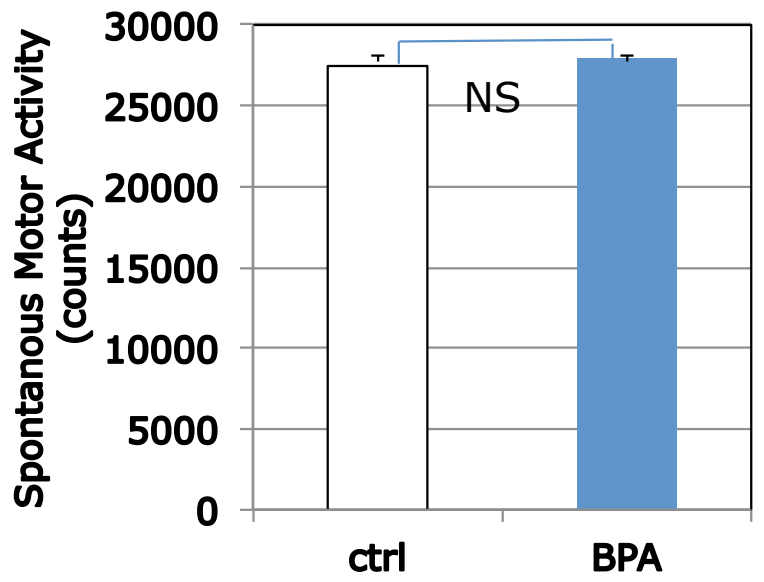

(B)
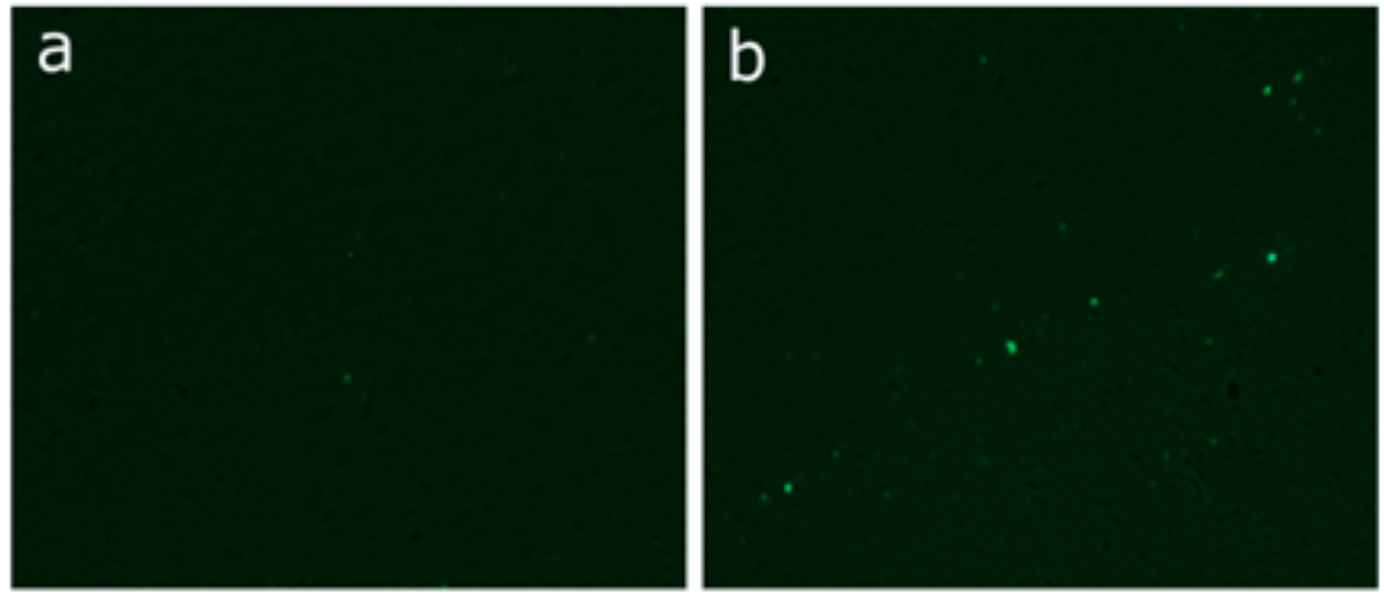

Fig. (6). Chronic exposure of adult rats to bisphenol A. Vehicle alone (ctrl) or bisphenol A (BPA) (3 mg/kg/day) was infused subcutaneously into adult Wistar rats using Alzet minipumps for 28 days. (A) After exposure, spontaneous motor activity was measured by a Supermex system. NS: Not significant. (B) Brains were then removed and subjected to Fluoro-Jade staining. a; control rats: b; bisphenol A-treated rats.

Recently, there have been some reports that examined adult exposure to bisphenol A. Particularly, it is of interest that bisphenol A exerts a powerful impact on neural system [14-16]. It was shown using a dose that is below the USEPA reference safe daily limit of $50 \mu \mathrm{g} / \mathrm{kg} / \mathrm{day}$ [31]. In this study, we employed $3 \mathrm{mg} / \mathrm{kg} /$ day, whose dose is as same as that used in rotenone models of Parkinson's disease [20]. Although the dose $(3 \mathrm{mg} / \mathrm{kg} /$ day $)$ is much higher than that of USEPA reference safe daily limit, it would be helpful to predict the low-dose effects of bisphenol A. Therefore, future study should be made sure by chronic exposure for much longer periods.

Dysfunction of dopamine neurons has been implicated in many neurological diseases, and they are especially vulnerable to degeneration, most notably in Parkinson's disease [9-11]. In this disease, motor disturbances do not present clinically until $\sim 80 \%$ of striatal dopamine has already been lost; however, other non-motor symptoms are evident before the onset of motor disturbances [9]. These include sleep abnormalities, autonomic dysfunction, anxiety, depression, gastrointestinal disturbances, and impaired cognition. Animal models of Parkinson's disease are generated by exposing adult animals to dopamine-selective neurotoxins, such as 6-OHDA or MPTP [12,13]. Non-motor phenotypes are present in these models [32]. Thus, it is tempting to extensively analyze bisphenol A models for similar manifestations of disease.

Parkinson's disease involves a progressive loss of dopamine neurons. Recently, to understand its etiology, attention has been paid to the developmental origins of health and disease (DOHaD) paradigm as a working hypothesis. The DOHaD paradigm was based on a concept by Dr. Barker [33], who hypothesized, after epidemiological studies, that fetal undernutrition during critical periods of vulnerability in early development leads to persistent changes in hormone levels and to altered tissue sensitivity to these hormones, permanently altering metabolism and body structure. His concepts thereafter extended to other adult diseases [34]. Many researchers further extended this concept to environmental issues. According to the $\mathrm{DOHaD}$ hypothesis, Parkinson's disease might begin early in life. One possible explanation for this phenomenon is that early exposure to neurotoxic chemicals reduces the number of dopaminergic neurons in the substantia nigra to levels below 
those needed to sustain normal function during the course of neuronal attrition associated with aging. The currently reported bisphenol A hyperactive rat model includes dopaminergic degeneration and hyperactive behavior that decreases to a normal level at adulthood, probably by compensatory input. Therefore, it may be an appropriate model to use to determine whether neonatal bisphenol A lesions might facilitate degeneration of dopaminergic neurons, leading to hypokinesia in adulthood, as seen in Parkinson's disease. Such distinct temporal effects are seen in von Economo's encephalic lethargica: hyperactivity, sleep disorders and antisocial personality disorder occur in childhood, and Parkinsonism is observed in adulthood [35], although the etiology remains unclear.

Some of recent human studies report that bisphenol A may affects humans, but others do not [36]. In the reports indicating the possible association of bisphenol A exposure with human diseases, the attention has been paid to altered behavior in children exposed to the chemical in utero or before puberty, as well as adult obesity, type- 2 diabetes, and cardiovascular disorders.

Thus, government regulation has been done to ban its use in baby bottles and sippy cups in some countries such as the United States, Canada, and EU.

In conclusion, acute and chronic exposure of adult rats to bisphenol A caused neurodegeneration in the substantia nigra. However, behavioral effects were not seen after either acute or chronic exposure to the chemical. The sensitivity of the central nervous system to bisphenol A was shown to be different at different life stages, confirming the greater vulnerability of the developing central nervous system.

\section{CONFLICT OF INTEREST}

The authors confirm that they do not have any conflict of interests concerning the content of this article.

\section{ACKNOWLEDGEMENTS}

This work was supported by NIES grant 12510 and a Grant-in-Aid for Scientific Research (KAKENHI) 24590774.

\section{REFERENCES}

[1] Vandenberg LN, Chahoud I, Heindel II, Padmanabhan V, Paumgarttten FJR, Schoenfelder G. Urinary, circulating and tissue biomonitering studies indicate widespread exposure to bisphenol A. Environ Health Perspect 2010; 118: 1055-70.

[2] Masuo Y, Ishido M. Neurotoxicity of endocrine disruptors: Possible involvement in brain development and neurodegeneration. J Toxicol Environ Health B Crit Rev 2011; 14: 346- 69.

[3] Castoldi AF, Patisaul HB, Aungst JL. Neurotoxic, neuroendocrine and neurobehavioural effects of bisphenol A. FAO/WHO Expert meeting on bisphenol A 2010.

[4] Ishido M, Masuo Y, Suzuki J, Oka S, Niki E, Morita EM. Dicyclohexylphthalate causes hyperactivity in the rat concomitantly with impairment of tyrosine hydroxylase immunoreactivity. J Neurochem 2004; 91: 69-76.

[5] Ishido M, Yonemoto J, Morita M. Mesencephalic neurodegeneration in the orally administered bisphenol A-caused hyperactive rats. Toxicol Lett 2007; 173: 66-72.

[6] Richter CA, Birnbaum LB, Farabollini F, et al. In vivo effects of bisphenol A in laboratory rodent studies. Reproduct Toxicol 2007; 24:199-224.

[7] Johnes DC, Miller GW. The effects of environmental neurotoxicants on the dopaminergic system: A possible role in drug addiction. Biochem Pharmacol 2008; 76: 569-81.
[8] Cooper JR, Bloom FE, Roth RH. In: The biochemical basis of neuropharmacology. $7^{\text {th }}$ ed. Newyork: Oxford Univ Press 2003, p. 405.

[9] Parkinson J. An essay on the shaking palsy. Whittingham and Rowland, London 1817.

[10] Strange PG. Parkinson's disease in brain biochemistry and brain disorders. New York: Oxford University Press 1992; pp. 161-86.

[11] Mizuno Y, Yoshino H, Ikebe S, et al. Mitochondrial dysfunction in Parkinson's disease. Ann Neurol 1998; 44: S99-S109.

[12] Dauer W, Przedborski S. Parkinson's disease: mechanisms and models. Neuron 2003; 39: 889-909.

[13] Blesa J, Phani S, Jackson-Lewis V, Przedborski S. Classic and new animal models of Parkinson's disease. J Biomed Biotech 2012; $1-10$.

[14] Leranth C, Szigeti-Buck K, MacLusky NJ, Hajszan T. Bisphenol A prevents the synaptogenic response to testosterone in the brain of adult male rats. Endocrinology 2008; 149; 988-94.

[15] Kim ME, Park HR, Gong EJ, Choi SY, Kim HS, Lee J. Exposure to bisphenol A appears to impair hippocampal neurogenesis and spatial learning and memory. Food Chem Toxicol 2011; 49: 33839.

[16] Eilam-Stock T, Serrano P, Frankfurt M, Luine V. Bisphenol A-impaires memory and reduces dendritic spine density in adult male rats. Behav Neurosci 2012; 126: 175-85.

[17] Masuo Y, Ishido M, Morita M, Oka S. Effects of neuronatal 6-hydroxydopamine lesion on the gene expression profile in young adult rats. Neurosci Lett 2002; 335: 124-8.

[18] Masuo Y, Pelaprat D, Montagne MN, Scherman D, Rostene W. Regulation of neurotensin-containing neurons in the rat striatum and substantia nigra. Effects of unilateral nigral lesion with 6-hydroxydopamine on neurotensin content and its binding site density. Brain Res 1990; 510: 203-10.

[19] Paxinos G, Watson C. The rat brain in stereotaxic coordinates, North Ryde: Academic Press Australia 1982.

[20] Panov A, Dikalov AS, Shalbuyeva N, Taylor G, Sherer T, Greenamyre JT. Rotenone model of Parkinson's disease: Multiple brain mitochondria dysfunctions after short term systemic rotenone intoxication. J Biol Chem 2006; 280: 42026-35.

[21] Luk KC, Kehm V, Carroll J, Zhang B, O'Brien P. Pathological $\alpha$-synuclein transmission initiates Parkinson-like neurodegeneration in nontransgenic mice. Science 2012; 338: 94953.

[22] Farabollini F, Porrini S, Della Seta D, Bianchi F, Dessi-Fulgheri F. Effects of perinatal exposure to bisphenol $\mathrm{A}$ on sociosexual behavior of female and male rats. Environ Health Perspect 2002; 110: 409-14.

[23] Kawai K, Nozaki T, Nishikata H, Aou S, Takai SM, Kubo C. Aggressive behavior and serum testosterone concentration during the maturation process of male mice: The effects of fetal exposure to bisphenol A. Environ Health Perspect 2003; 111: 175-8.

[24] Farabollini F, Porrini S, Dessi-Fulgheri F. Perinatal exposure to the estrogenic pollutant bisphenol A affects behavior in male and female rats. Pharmacol Biochem Behav 1999; 64: 687-94.

[25] Aloisi AM, Della Seta D, Rendo C, Ceccarelli I, Scaramuzzino IA, Farabollini F. Exposure to the estrogenic pollutant bisphenol A affects pain behavior induced by subcutaneous formalin injection in male and female rats. Brain Res 2002; 937: 1-7.

[26] Adriani W, Della-Seta D, Dessi-Fulgheri F, Farabollini F, Laviola G. Altered profiles of spontaneous novelty seeking, impulsive behavior, and response to D-amphetamine in rats perinatally exposed to bisphenol A. Environ Health Perspect 2003; 111: 395401.

[27] Kubo K, Arai O, Omura M, Watanabe R, Ogata R, Aou RS. Low dose effects of bisphenol A on sexual differentiation of the brain and behavior in rats. Neurosci Res 2003; 45: 345-56.

[28] Fujimoto T, Kubo K, Aou S. Prenatal exposure to bisphenol A impairs sexual differentiation of exploratory behavior and increases depression-like behavior in rats. Brain Res 2006; 1068: 49-55.

[29] Nakamura K, Itoh K, Yaoi T, Fujiwara Y, Sugimoto T. Murine neocortical histogenesis is perturbed by prenatal exposure to low doses of bisphenol A. J Neurosci Res 2006; 84: 1197-205.

[30] Ishido M, Masuo Y, Terasaki M, Morita M. Rat hyperactivity by bisphenol A, but not by its derivatives, 3-hydroxybisphenol A or bisphenol A 3,4-quinone. Toxicol Lett 2011; 206: 300-5.

[31] USEPA Integrated Risk Information System. Bisphenol A, CASRN 80-05-7 (1993). 
[32] Vernon AC. Mice with reduced vesicular monoamine storage content display nonmotor features of Parkinson's disease. J Neurosci 2009; 29: 1284-844.

[33] Bateson P, Barker D, Clutton-Brock T, et al. Developmental plasticity and human health. Nature 2004; 430: 419-21.

[34] Gilman MW, Barker D, Bier D, et al. Meeting report on the $3^{\text {rd }}$ international congress on developmental origins of health and disease (DOHaD). Pediatr Res 2007; 61: 625-9.
[35] von Economo C. Encephalitis lethargica. In: Newman KO, Ed. Its sequelae and treatment. London: Oxford University Press 1931; pp. 2-3.

[36] Rochester JR. Bisphenol A and human health: A review of the literature. Reprod Toxicol 2013; 42: 132-55.

(C) Ishido and Masuo; Licensee Bentham Open

This is an open access article licensed under the terms of the Creative Commons Attribution Non-Commercial License (http: //creativecommons.org/licenses/by$\mathrm{nc} / 3.0 /$ ) which permits unrestricted, non-commercial use, distribution and reproduction in any medium, provided the work is properly cited. 